H. Dingler, Philosophie der Logik und Arithmetik. E. Reinhardt, München 1931. Preis geh. RM 7,50.

Das Buch beschäftigt sich hauptsächlich mit dem Wesen der mathematischen Kalküle. Zur Aufstellung und Handhabung derselben sind nach Ansicht des Verf. gewisse, nicht weiter analysierbare "Grundfähigkeiten" erforderlich, die als "aktive Logik" dem Kalkül selbst als "passiver Logik" gegenübergestellt werden (ungefähr entsprechend der Hilbertschen Unterscheidung von Metamathematik und Mathematik). Art und Verwendungsweise der Grundfähigkeiten-(besonders zum Aufbau der Arithmetik) werden auseinandergesetzt und versehiedene erkenntnistheoretische Fragen von diesem Gesichtspunkte aus behandelt. Als konkrete Anwendung der allgemeinen Betrachtungen folgt eine Untersuchung uber ein formales System der Arithmetik, welche den von Hilbert gesuchten Widerspruchfreiheitsbeweis liefern soll, in Wahrheit aber bestenfalls die triviale Tatsache der Widerspruchsfreiheit der elementaren Rechenregeln (distributives Gesetz etc.) zeigen könnte.

K. Gödel.

H. Dingler, Das System. Das philosophisch-physikalische Grundproblem und die exakte Methode der Philosophie. Reinhardt, München 1930. Preis brosch. RM 5,50, geb. 7,80

Das hier zu besprechende Werk Dinglers will eine genaue Erläuterung des von ihm geschaffenen Begriffs der reinen Synthese geben. Zu diesem Zweck behandelt er eingehend das Wesen des "Systems". Das System ist eine Reihe von Sätzen, die derart angeordnet sind, dab die jeweils späteren ans den vorhergehenden deduziert werden können (29). Es soll das vollkommene System der Wissenschaft sein, da ihm die "Realgeltung vollkommen und in apodiktischer Form und mit absoluter Genauigkeit" gesichert sein soll (35). Es ist klar, daß, um dieser Folgerung zu genügen, die Sätze dieses Systems nicht Aussagen über die un ja nie völlig. bekannte "struktur" der Welt sein können. Nach Dingler ist die Welt vielmehr völlig strukturlos, ohne "Beschaffenheit" (127) und die Sätze des Systems Handlungsanweisungen zum Bau unserer Apparate, "praktische Maßnahmen unsererseits zum Zwecke der nutzhaften Bëwältigung der Realität". Durch die Beschaffenheit unserer Apparate prägen wir der völlig plastischen Welt erst eine Gesetzlichkeit auf, die begründet ist in unserem freien Willen, der, um die Danererhaltung der Menschbeit zu begründen (43), gemäß dem „Prinzip der Einfachheit" das System begründet.

Das klar geschriebene Buch ist in teressant als konsequente Zuendeführung des Konventionalismus, der ja Entscheidendes zur philosophischen Durchleuchtung der exakten Wissenschaften geleistet hat. In der Überspitzung, die er bei Dingler erfährt und die zur Ablehnung von Relativitäts- und Quantentheorie zugunsten der New to n schen Physik führt, scheint er sich allerdings überschlagen und in einem wirklichkeitsfremden Paradoxon totgelaufen zu haben.

\title{
H. Neider.
}

F. Kuntze, Von den neuen Denkmitteln in der Philosophie. Heft 3. Der Begriff der Elemente des Gedankens in der Mathematik. $33 \mathrm{~S}$. Winter, Heidelberg 1928. Preis RM 1,-.

F. Kuntze, Der morphologische Idealismus. Seine Grundlagen und seine Bedeutung. (VIII +116 S.) C. H. Becksche Verlagsbuchbandlung, München. Preis RM 6,-

Die zuerst genannte kleine Schrift verfolgt pädagogische Ziele. Sie vermittelt dem Anfänger die Bekanntschaft mit den Grundbegriffen der nichteukli- 